\title{
Pharmacodynamic Modelling of Biomarker Data in Oncology
}

\author{
Robert C. Jackson \\ Pharmacometrics Ltd., Whittlesford, Cambridge CB22 4NZ, UK \\ Correspondence should be addressed to Robert C. Jackson, rjackson1943@aol.com
}

Received 5 November 2011; Accepted 6 December 2011

Academic Editors: S. Cuzzocrea and E. M. Urbanska

Copyright (C) 2012 Robert C. Jackson. This is an open access article distributed under the Creative Commons Attribution License, which permits unrestricted use, distribution, and reproduction in any medium, provided the original work is properly cited.

\begin{abstract}
The development of pharmacodynamic (PD) biomarkers in oncology has implications for design of clinical protocols from preclinical data and for predicting clinical outcomes from early clinical data. Two classes of biomarkers have received particular attention. Phosphoproteins in biopsy samples are markers of inhibition of signalling pathways, target sites for many novel agents. Biomarkers of apoptosis in plasma can measure tumour cell killing by drugs in phase I clinical trials. The predictive power of PD biomarkers is enhanced by data modelling. With pharmacokinetic models, PD models form PK/PD models that predict the time course both of drug concentration and drug effects. If biomarkers of drug toxicity are also measured, the models can predict drug selectivity as well as efficacy. PK/PD models, in conjunction with disease models, make possible virtual clinical trials, in which multiple trial designs are assessed in silico, so the optimal trial design can be selected for experimental evaluation.
\end{abstract}

\section{Introduction}

The well-known limitations of cancer chemotherapy are its toxicity to normal tissues (a reflection of limited selectivity), its low clinical response rates (often $20 \%$ or less), and the narrow clinical spectrum of existing drugs. The toxicity to normal tissues of classical cytotoxic agents reflects the fact that these drugs target processes central to division of all cells, whether normal or transformed, such as DNA replication, RNA transcription, or microtubule function. The newer "targeted agents" designed to exploit specific molecular lesions in individual cancers are indeed less toxic to normal tissues than are cytotoxic drugs, but this greater therapeutic index has been achieved at the cost of a narrower antitumour spectrum. This narrow spectrum is being addressed by "stratified medicine," that is, by matching the treatment to the presence in a tumour, or to the expression level, of the molecular target against which the targeted agent is directed. For example, if an experimental drug has been shown to be selectively active against cells with mutant, constitutively activated K-ras, a stratified clinical trial would treat patients whose tumours expressed that mutation as a separate group, This approach is statistically powerful, but can lead to complex clinical trial designs: for example, if treatment is to be stratified according to expression of three biomarkers, for example, wild-type versus mutant K-ras, wild-type versus mutant p53, and normal versus mutant B-raf, this would require an eight-arm trial. In practice, human tumours may carry large numbers of genetic abnormalities - often fifty or more-any of which could influence drug response. It is thus argued that ultimately, to optimise the treatment of a particular patient will require "personalised medicine," in which treatment will be determined by the expression of a large panel of biomarkers, ultimately, perhaps, by a whole genome mRNA expression analysis. Some commentators distinguish between "predictive" biomarkers, that can predict response of cells to treatment at the level of cell biology or biochemical pharmacology, and the more restricted class of "prognostic" markers, which can be related to clinical outcome (as, e.g., expression of estrogen receptor in breast cancer predicts for clinical response to tamoxifen). A cellular or molecular response to treatment does not guarantee a clinical response, but without a molecular response clearly there cannot be a clinical response.

In contrast to predictive biomarkers, which attempt to predict from the properties of the tumour whether it is likely to respond to a particular treatment, pharmacodynamic (PD) biomarkers provide a measure, posttreatment, of whether the drug has reached its target and exerted a pharmacological response, and if so, what was the degree of 
response [1-3]. Once again, a PD response is no guarantee of a significant clinical response, but without a pharmacological response, we would not expect to see a clinical response. PD biomarkers can thus be used to make a decision on whether to continue treatment, to stop treatment, or to switch to a different treatment. Currently, the clinical application of PD biomarkers is confined to this kind of qualitative decision-making. If we want to use PD biomarkers to make quantitative decisions, for example, to adjust the dose, or change the schedule of administration, a PK/PD model is the appropriate tool. The first review of PD modelling of biomarker data in oncology was published recently [4], and the authors commented on the small number of reports in the literature. However, biomarker measurements are becoming standard in phase I clinical studies, and investigators are increasingly starting to fit their biomarker data to PD models. The next few years will see PD modelling of biomarker data become as widely used as PK modelling of drug concentrations.

\section{Current Uses of PD Biomarkers}

Historically, phase I clinical trials in oncology used a starting dose that was expected to be safe, based upon toxicology in two animal species. The dose in the phase I patients would then be escalated until a dose-limiting toxicity was identified. A phase II clinical trial would be designed based upon a dose and schedule of administration that was tolerated in phase I. This approach had several limitations: it provided no estimate of what the target phase I maximum tolerated dose (MTD) was likely to be, and it gave no clue as to whether the dose and schedule taken into phase II was likely to be therapeutically effective. A majority of the phase I patients were exposed to doses that were too low to have any chance of being active. These limitations have been partially surmounted through the use of PK modelling. Preclinical PK studies in the same species used for preclinical antitumour studies (usually mice, sometimes rats) made it possible to correlate antitumour responses with PK. Plasma concentrations achieved in the test species could be compared with concentrations known to have in vitro activity. Preclinical PK studies would also give an estimate of oral bioavailability. The peak plasma level and AUC that correlated with preclinical antitumour activity gave an indication of the dose to be aimed for in the phase I clinical trial, reducing the number of dose escalations needed and minimising the number of patients exposed to subtherapeutic doses. The power of preclinical data could be enhanced by fitting the data to a PK model. This made it possible to use mouse or rat data to predict PK in other species (including humans). PK models can be used to predict optimal dosing schedules, by relating plasma clearance or plasma half-life to different dosing intervals. Such is the power of PK modelling that it would be unthinkable to take an investigational oncology drug into clinical trials without having fitted preclinical data to a PK model, and without then obtaining clinical PK data. The preclinical model is usually adapted to predict clinical PK for different routes and schedules of administration, in patients of different body weights, and different degrees of kidney or liver function.

For all its advantages, the predictive power of PK modelling is limited. Its most conspicuous limitation is that it is normally based upon plasma PK, while the therapeutically relevant drug concentration is that in the tumour, and the toxicologically relevant drug concentration is that in the normal tissue which is the site of dose-limiting toxicity. These concentrations can sometimes be obtained in preclinical studies, but almost never in phase I clinical studies, since they would require frequent, multiple biopsies of tumour and normal tissues. The usefulness of plasma PK is also limited by the fact that many anticancer drugs, including all the nucleoside antimetabolites, are actually prodrugs. The cellular concentrations of their active nucleotide species have a complex and indirect relationship to the plasma concentrations of their nucleoside precursors. The concentration dependence of drug-target interactions may vary widely between species, which complicates preclinical-clinical correlations. This can be partially mitigated by doing preclinical studies against human tumour xenografts in immunedeficient mice. However, the normal tissues are still those of mice, making it impossible to draw firm conclusions about drug selectivity. Another limitation is that antiumour effects are normally timedependent, as well as dosedependent. For example, many anticancer agents induce caspase-dependent apoptosis, a process that lags many hours behind the critical drug concentration that induced it, and drug exposures, even above the critical concentration, that last for less than the critical exposure time, produce only transient, reversible effects, rather than cell death. Classical PK modelling cannot describe these complex dose-time relationships. Indirect PK models have been used to describe antitumour drug effects [5] and these models can describe systems in which the effect lags the drug concentration. However, the time-dependence of antitumour drug effects is frequently a function of the cytokinetic properties of the tumour, and these often differ extensively between mouse and human tumours.

Pharmacodynamic (PD) endpoints address all these limitations. By measuring a drug effect at the tumour site (or at the site of a toxic drug effect), they provide direct evidence that the drug has reached its target, and they provide a quantitative measure of the degree of drug response and (given sufficiently frequent sampling) of the time dependence of the drug response. Currently, most PD biomarkers are measured in biopsy material, which imposes limitations on their clinical use, but plasma biomarkers and noninvasive imaging biomarkers are becoming increasingly used. Preclinical PD biomarker data can assist in phase I clinical trial design. By comparing a PD biomarker response with an antitumour response in mice, it is possible to establish what degree of biomarker response will predict for a clinical response. An increasing number of oncology phase I clinical trials are supplementing clinical and toxicological endpoints with PD biomarker endpoints. In this way, biomarkers can assist in dose-ranging in phase I studies. If a biomarker reaches an optimal endpoint before dose-limiting toxicity is seen, this may indicate that it is not necessary or desirable to treat patients at or near an MTD, as has been 
customary in oncology. As a long-term objective, it should be possible to validate PD biomarkers as surrogate efficacy endpoints. This has been done in other therapeutic areas, but not yet in oncology.

Although the use of PD biomarkers in preclinical anticancer drug development has become very common and biomarker use is becoming more frequent in phase I clinical trials, very few investigators have fitted their PD data to a PD model [4]. It would be almost unthinkable to measure PK data and not model it, because modelling maximises the information content and predictive power of the data. The qualitative or semiquantitative uses of PD biomarkers discussed above have established that knowing the PD effects of anticancer drugs can provide important insights. So why is greater use not made of PD modelling? Probably because it is more difficult than PK modelling. It is instructive to consider why this is, and what can be done about it.

\section{Differences between PK and PD Modelling}

PK modelling is a generic technology, that is, the same techniques, the same equations, and the same software can be used across all therapeutic areas. PD modelling, by contrast, is likely to be specific to a particular therapeutic area, or even, depending upon the exact PD biomarker employed, to a particular drug target, or drug class. This obviously increases the difficulty, the cost, and time commitment of developing a new PK/PD model. Because PD models are more drugspecific, they tend to be more mathematically complex and more computationally intensive. The parameterization and validation are likely to be more experimentally intensive.

The experimental methods used to obtain PK data are also generic across all therapeutic areas, and currently almost always use the powerful analytical technique of liquid chromatography with mass spectrometric detection (LC/MS). In contrast, PD endpoints use a wide range of analytical techniques, and again will usually require new analytical method development for each endpoint.

PD modelling necessarily requires describing events at the drug target site. For some anticancer drugs (e.g., therapeutic antibodies), the target sites are extracellular, and assuming that concentrations in the extracellular fluid closely mirror plasma concentrations may be a reasonable approximation for such drugs. However, most anticancer drugs act at intracellular sites. While several detailed physiologically based PK models have been described that accurately predict drug levels in tumour and normal tissues, such models are usually developed long after the drug has been through early clinical trials. The PK models that guide phase I and phase II clinical trial design are almost invariably based upon plasma PK.

\section{Techniques of PD Modelling in Oncology}

4.1. PD Models and PK/PD Models. PD models are frequently developed and validated in vitro, using constant drug concentrations. In such conditions, a pharmacodynamic response can be related directly to drug concentration. In vivo, with a constantly changing drug concentration, the pharmacodynamics and pharmacokinetics are inextricably linked. It is, of course, possible to relate a biomarker PD effect to an administered drug dose without explicitly modelling drug concentrations. However, in practice, PK data and a PK model are usually available, so that PD modelling studies in the in vivo and clinical situation generally imply a $\mathrm{PK} / \mathrm{PD}$ model.

4.2. Direct $P K / P D$ Models. The simplest form of $\mathrm{PK} / \mathrm{PD}$ model consists of a dose-response equation coupled to a PK model. Usually the dose-response equation will be a Hill equation, often referred to by pharmacologists as the Emax equation. This is a three-parameter equation, with parameters describing the maximal effect (Emax), the concentration of drug that gives a $50 \%$ maximal effect (IC50), and the slope, $m$ (the Hill coefficient). If $m=1$, the dose-response curve is a rectangular hyperbola. When $m>$ 1 , the dose-response curve is steeper and sigmoidal, with an inflection point at a drug concentration of IC50. When $m<$ 1 , the dose-response curve is more shallow. In some cases, maximal drug concentrations do not completely reduce the biological response to zero, but give a plateau. These doseresponse curves can be described by a four-parameter version of the Hill equation, in which the fourth parameter is the plateau value of the effect.

Equations other than the Hill equation have been used in direct PK/PD models [6]. For irreversible inhibitors, or reversible inhibitors with very slow "off" rates, the doseresponse relationship may be approximately linear. Killing of bacteria by antibiotics has been described by logarithmic dose-response curves [6], and some receptor ligands show biphasic (bell-shaped) dose-response curves, in which the drug effect reaches a maximal value and then decreases with further increase in drug concentration.

Some anticancer drugs may affect biochemical pathways with relaxation times that are very short in comparison with drug clearance times. In such cases, if the PD biomarker being measured reflects a direct product of the inhibited reaction, the pharmacodynamics may track the drug concentration closely in time. Some protein phosphorylation biomarkers may fall into this category. The anticancer thymidylate synthase inhibitor, Thymitaq (AG337), enters and exits cells very rapidly, and its inhibitory effects on thymidylate synthase are immediate. The phase I clinical trial used circulating deoxyuridine as a measure of thymidylate synthase inhibition [7], and the kinetics of the system are such that this plasma biomarker tracks a direct $\mathrm{PK} / \mathrm{PD}$ relationship.

4.3. Indirect $P K / P D$ Models. Direct $P K$ models describe the situation where the drug effect is immediate, so that the PD effect directly tracks the drug concentration. For many drugs, the effect is a function of both concentration and time, and for such drugs the total effect may be proportional to $C \times t$ (where $C$ is concentration and $t$ is time). In PK terminology, for such drugs the effect is proportional to AUC. Kalns 
et al. [8] noted that a more generally applicable relationship is given by

$$
s=C^{n} \times t
$$

where $s$ denotes drug sensitivity for a particular system and $n$ is a pharmacodynamic exponent that relates the relative importance of concentration and time in determining drug effects. When $n>1$, concentration is the primary determinant of the drug effect, and when $n<1$ the effect is primarily time dependent. Kalns et al. suggested that the value of the $n$ parameter had implications for selection of optimal clinical dosage regimens. For instance, when $n>1$, bolus administration should be more effective than an infusion. The $s$ parameter may be obtained from experimental data by either a two-step process in which IC50 values are obtained for a range of exposure times, and the IC50 estimates (representing $C$ ) are fitted to the equation of Millenbaugh et al., or, alternatively, the data values for all time points are fitted by nonlinear regression to

$$
f=\frac{C^{m}}{\left[(s / t)^{m / n}+C^{m}\right]},
$$

where $f$ is the fraction affected, $m$ is the Hill coefficient, and $C, t, n$, and $s$ are as defined for the equation of Millenbaugh et al. $[6,8]$.

Sharma and Jusko [5] considered four kinds of indirect $\mathrm{PD}$ relationship. If a PD effect is mediated by accumulation of a particular metabolite or ligand, and the drug effect inhibits or accelerates that accumulation, then the drug will have antagonistic or agonistic effects, respectively, in that system. If the PD effect is mediated by depletion of a metabolite or ligand, a drug that inhibits or accelerates that depletion will again have antagonistic or agonistic effects. Sharma and Jusko derived equations to describe these four kinds of indirect PD effect [5]. Jordan and Gieschke [9] derived alternative, explicit forms of these relationships and published a computer program for solving them. Most anticancer drugs exert their effects after a protracted time delay, so that indirect PD models are usually the most appropriate description. A recent example of PK/PD modelling of a biomarker response that used an indirect PD model is the work of Choo et al. [10], studying, the Genentech allosteric MEK inhibitor, G-573. In this case the biomarker was the immediate reaction product, phospho-ERK, measured in tumour xenograft tissue.

Two kinds of indirect PD effect are frequently seen with anticancer drugs. Many drugs cause cell cycle perturbations, causing, for example, cell cycle arrest specifically in a particular cell cycle phase. For example, compounds that cause DNA strand breaks will trigger DNA damage responses, which arrest cell cycle progression of cells in G1 phase and S phase. Drugs that inhibit tubulin polymerisation or depolymerisation cause cell cycle arrest in M phase. These drugs will only affect cells that are in the sensitive phase of the cell cycle, so to exert their maximal effect they must be present for a significant fraction of the cell cycle time, which for human tumour cells is often about 24 hours.
Many anticancer drugs kill tumour cells by inducing caspasedependent apoptosis, a process that typically takes about 5-24 hours. These two kinds of indirect PD effect are so important in oncology that specialised PD models have been developed to describe their effects, which are considered below.

4.4. Cytokinetics-Based PD Modelling. Cytokinetics is the quantitative description of the progression of cells through the cell division cycle. Cells are characterised by the cell cycle time (the time required for a newly replicated cell to progress through a complete round of growth and replication) and by the time the cell spends in each of the phases of the cell cycle: G1 phase, in which a newly replicated cell doubles in size; $S$ phase, in which the cell doubles its DNA content; G2 phase, in which the replicated DNA is deconvoluted and prepared for packaging into chromosomes; $M$ (mitosis) phase, in which the replicated chromosomes are sorted into two complete sets and moved to opposite poles of the cell, prior to the act of cytokinesis, in which one tetraploid cell becomes two diploid sells. Other important cytokinetic parameters are the size of the G0 fraction, cells that move reversibly from G1 phase into a noncycling state, the cell loss factor, that is, the fraction of newly replicated cells that are defective and unable to continue replicating, and the fraction of G1 cells that irreversibly leave the cell cycle to become differentiated or senescent cells. These cytokinetic transitions are controlled by a complex group of positive and negative growth factors, and their associated signal transduction pathways. Internal regulation of the cell cycle is through a number of cell cycle checkpoints. For example, cells that have not reached a critical size or have not activated the transcription factors required for generate DNA precursors are unable to progress from G1 into $S$ phase (the G1 checkpoint). Mitotic cells that have not correctly sorted their replicated chromosomes into two equal sets are unable to proceed to cell division (the spindle assembly checkpoint). Cells with DNA damage (strand breaks, cross-links, or apurinic or apyrimidinic sites) are unable to continue DNA replication (DNA damage checkpoint).

Cancer is a disease of cell cycle regulation, specifically, it is a disease of cell cycle checkpoints. All tumour cells have a dysfunctional G1 checkpoint, either because of mutation, deletion, or epigenetic silencing of checkpoint components such as p53, p16, or p21, or because of overexpressed or constitutively activated growth factor receptors or signalling proteins (e.g., ras) which result in override of the G1 checkpoint $[11,12]$. All cancer cells are aneuploid [13]. This is either because of defects in the spindle assembly checkpoint, which means that cells with incorrectly sorted chromosomes proceed to cell division before they are ready, or because cells with a defective DNA damage response allow cell division to occur before DNA damage has been repaired.

Consistent with this view of cancer as a disease of two or more cell cycle checkpoints, almost all anticancer drugs act on control of the cell cycle. Cytotoxic anticancer agents typically inhibit DNA synthesis or cause DNA damage, or block microtubule function, and modern targeted agents 
typically block growth factor signalling, inhibit checkpoint components, or trigger apoptosis, selectively in cells with aberrant checkpoint function.

Two kinds of biomarkers of cell cycle perturbation are used. It is possible to determine the proportion of cells in different phases of the cell cycle, for example, by flow cytometry, and this can be used as a PD biomarker for drugs that cause cell cycle-specific effects. Alternatively, proteins that are expressed specifically at particular stages of the cell cycle, or which are phosphorylated at certain points in the cycle, can be measured by immunoassay or western blotting. Models of the cell cycle have been reported that describe the process in terms of cytokinetic parameters, and with varying amounts of molecular detail $[6,14-17]$. These models can be used to describe PD effects on both cytokinetic and molecular biomarkers.

4.5. Models of Signal Transduction Pathways. These pathways, which form a link between growth factor and cytokine receptors on the cell surface, and transcription factors in the nucleus, are targets for many anticancer drugs. The pathway components include G-proteins, protein kinases, and accessory proteins for transcription factors. The control of these pathways is complex, involving multiple positive and negative feedback, convergent and divergent branching, and cross-talk between pathways [18]. Detailed models have been published for the EGF signalling pathway $[19,20]$ and the Wnt signalling pathway [21], whose components are often mutated or upregulated in cancer cells.

4.6. Models of Apoptosis. Effective anticancer drugs cause selective killing of tumour cells - transient, reversible cell stasis or growth delay typically do not confer much therapeutic benefit, though non-cytotoxic drugs that block metastasis, or tumour angiogenesis, or stimulate antitumour immunity are an important growth area in oncology. Biomarkers are available both for apoptotic cell death and for noncaspasemediated cell death pathways such as necrosis. These will be discussed below. Fussenegger et al. [22] reported a kinetic model of apoptosis that accurately described the process of caspase activation, including the prolonged reversible stage before the irreversible production of active caspase-3. This approach was extended and further validated by Hua et al. [23] and Bentele et al. [24]. Their models have been applied to PD modelling of anticancer drug PD, and experimental examples will be discussed below.

4.7. Complex Dynamic System Models for PD Modelling. The pharmacodynamics of anticancer drugs is particularly complex because they often require describing processes at several levels of biological organization. For example, the effects of an antiangiogenic drug may involve the VEGF receptors, their associated signalling pathways, replication and migration of endothelial cells, and effects on blood supply to tumours and normal tissues. Similarly, the essential dynamics of an antimetastatic drug involve effects on biochemical pathways, on cells, and on cell distribution among multiple tissues. The PD of antiangiogenic or antimetastatic drugs require, minimally, a modelling approach that represents these hierarchical systems $[25,26]$. These hierarchical models have generally been fitted to biological data (numbers of blood vessels, numbers of metastatic cells), but in principle these hierarchical models have the potential to describe the PD of molecular biomarkers. Eissing et al. [27] have described a software platform for integrating PK, wholebody physiology, disease biology and molecular reaction networks. It is intended that this platform can be used to develop models than can test hypotheses involving pharmacogenomics, drug-drug interactions, and drug-metabolite interactions. A prototype model based upon this system simulated the progression of a pancreatic tumour, and its response to a prodrug of a Raf kinase inhibitor. The model described signal transduction, cell cycle progression, and proliferation. A virtual clinical trial modelled treatment outcomes in a genetically heterogeneous population [27].

\section{Examples of PD Modelling of Biomarker Data}

5.1. Plasma Biochemical Markers. One of the first plasma PD biomarkers of an anticancer drug to be used clinically was circulating deoxyuridine in patients treated with the thymidylate synthase inhibitor, Thymitaq (AG337) [7]. The biomarker data from that study were not modelled, but PD models exist that can be used to model this data [28].

5.2. Cytokinetic Markers. Cytokinetic data, mainly obtained from flow cytometry, have formed the basis for a number of PD modelling studies. Basse et al. [29] developed a cytokinetic model of the melanoma cell line NZM13. The model was used to study effects of paclitaxel on cytokinetic properties of the cells, including rate of entry into mitosis and rate of drug-induced DNA degradation. The model was subsequently used to study radiation-induced changes to five human melanoma cell lines [30]. Irradiation at 9 Gy caused G2 phase arrest in all lines for at least $96 \mathrm{hr}$. Two cell lines with wild-type p53 also exhibited G1 arrest with recovery over $15 \mathrm{hr}$, as well as evidence of cell loss. Resumption of cycling of surviving cells was consistent with the results of clonogenic assays. Circulating tumour cells have been used as a PD biomarker in lung cancer patients [31]. In nasopharyngeal carcinoma, a tumour linked with EpsteinBarr virus infection of the malignant cells, circulating viral load, has been used as a PD biomarker [32].

5.3. Protein Phosphorylation Markers. Since many targeted anticancer agents act by inhibiting protein kinases in signalling pathways, measurement of the phosphoproteins that are products of these reactions has become an important source of PD biomarkers in tumour biopsies [33]. Phosphoprotein biomarkers have been developed for inhibitors of Bcr-abl [34], c-Met kinase [35], MEK [10, 36], PI-3 kinase [37], and VEGF-R2 tyrosine kinase, and these biomarker studies formed the basis for PD modelling. These studies have been used to relate the extent of pathway inhibition to tumour response in mice, to suggest clinical doses based 
upon preclinical data and to identify optimal combinations of targeted agents [33].

5.4. Spindle Checkpoint Biomarkers. Many important anticancer drugs, including vinca alkaloids and taxanes, act by blocking mitosis. Prolonged mitotic arrest may be followed by apoptosis or by escape of the arrested cells back into the cell cycle. Cells in which mitosis has been disrupted may undergo abnormal cell division, resulting in polyploidy or aneuploidy. Several newer experimental drugs inhibit components of the mitotic apparatus, including kinesin spindle proteins, aurora kinase A, aurora kinase B, pololike kinase 1, and NEK-2. It is believed that the anticancer selectivity of drugs that block mitosis arises from the fact that tumour cells frequently have a defective mitotic spindle assembly checkpoint (SAC). This checkpoint acts to prevent cells entering anaphase (in which pairs of replicated chromosomes separate, with one member of each pair migrating to opposite poles of the dividing cell), until the chromosomes are correctly attached. The SAC ensures that each daughter cell receives one, and only one, copy of each replicated chromosome.

Kinetic models of the SAC have been described that include the site of action of aurora kinase B [38-40], using phosphorylation of histone $\mathrm{H} 3$ as a biomarker. Inhibition of aurora kinase B decreases histone $\mathrm{H} 3$ phosphorylation $[39,41]$. Polyploidy was also used as a cytogenetic biomarker for aurora kinase B inhibition [41]. The model was later extended to include the site of action of aurora kinase A [42]. Biomarkers of aurora kinase A inhibition include mitotic arrest, increased phosphorylation of histone $\mathrm{H} 3$, and decreased aurora kinase A autophosphorylation. This model was used as the PD component of a PK/PD model [43] which was used to describe biomarker dynamics in tumour-bearing mice treated with the aurora kinase A/B inhibitor CYC116 [41].

5.5. Toxicity Biomarkers. Ideally cancer treatment should be monitored by measuring toxicity biomarkers as well as tumour response biomarkers, in order to quantify selectivity as well as efficacy. There have been very few published studies of this kind. Lindauer et al. [44], in a study of sunitinib, measured plasma levels of VEGF-A and VEGF-C and of soluble VEGF-receptor 2 as PD biomarkers in healthy volunteers, as well as plasma PK. Simultaneously, blood pressure was measured as a toxicity marker. Greystoke et al. reported a clinical biomarker study in which nucleosomal DNA and CK18 were measured as biomarkers of apoptosis, and FLT3 ligand was measured as a biomarker of myelosuppression in lymphoma patients undergoing chemotherapy [45]. These biomarkers are now undergoing large-scale validation in a multicentre phase III study.

5.6. Biomarkers of Apoptosis. Apoptosis biomarkers have been a subject of great interest in oncology, because induction of apoptosis represents the final downstream event in the action of many, perhaps most, anticancer drugs. In preclinical systems, many of the molecular events in this process have been tracked in detail as a function of time: caspase- 3 activation, cleavage of poly-ADP ribose polymerase (PARP), and terminal dUTP nick end labelling (TUNEL), a measure of DNA fragmentation. After treatment of tumour cells with apoptosis-inducing drugs, flow cytometric studies generally show accumulation of cells with a sub-G1 DNA content, and this has been widely used as a cell death biomarker. By examining activation of intermediates in the caspase cascade, it is possible to distinguish between the type I apoptosis pathway (triggered by activation of cell membrane death receptors (e.g., receptors for TNF, FAS, and TRAIL) and the type II pathway (triggered by mitochondrial damage)). For example, activated caspase- 8 and caspase- 10 have been used as biomarkers for type I apoptosis, and activated caspase-9 and Bcl-2 have been used as specific biomarkers for type II apoptosis [23]. The two pathways converge on a common downstream effector, caspase-3, which may be used as a biomarker for total apoptosis. Hua et al. [23] modelled both pathways and validated their model against experimental data from Jurkat human T cells. They used their model to study the kinetics of death signalling by FAS ligand.

Biomarkers of apoptosis have a great advantage over most of the other PD biomarkers of anticancer drug action that have been studied. Whereas most biomarkers are specific for the action of drugs acting on a particular target site, or at best a particular target pathway, almost all anticancer drugs ultimately induce apoptosis, so these markers may be regarded as generic (drug-independent) markers of tumour cell killing. Some of the apoptosis markers most widely used in preclinical studies are intracellular (caspase activation, PARP cleavage) or cellular (sub-G1 DNA content), and thus require biopsy material. This limits their clinical applicability. For clinical application, attention has focussed on plasma biomarkers of apoptosis, which are regarded as minimally invasive (i.e., they just require a drop of blood). Potential plasma biomarkers for apoptosis that have been assessed include $\alpha$-fetoprotein [46], $\beta$-human chorionic gonadotrophin [46], lactate dehydrogenase [31, 46], and nucleosomal DNA [45, 47]. However, most attention has been focussed on plasma levels of cytokeratin-18 (CK18) and caspase cleavage fragments of CK18 [48]. Linder and colleagues [48-50] and Barak et al. [51] pointed out that cytokeratins are expressed primarily in epithelial tissues. When the epithelial cells of the skin die, their contents are sloughed outside the body. When the epithelial cells of the intestine die, their contents are sloughed into the intestinal lumen. Thus, only low baseline levels of cytokeratins or cytokeratin fragments are normally seen in plasma. However, dying epithelial tumour cells release cytokeratins into the blood. Cancer patients frequently have elevated levels of circulating cytokeratins, which has been attributed to release from spontaneously necrotic or apoptotic tumour cells [49]. Research in this area was facilitated by the availability of antibodies against CK18, termed M65 [52] or TPS [51]. Another antibody, M30, recognises a neoepitope on a fragment of CK18 specifically generated by caspase cleavage. ELISA assays bave been developed based upon the M30 and M65 antibodies, which are commercially available (Peviva $\mathrm{AB}$, Bromma, Sweden). It is thus argued that M30 gives a 
measure of apoptotic cell death and M65 gives a measure of total epithelial cell death, including necrosis [49, 52]. Cummings et al. [53] showed that an antisense compound (AEG35156) targeted against the antiapoptotic protein XIAP caused elevations of the M30 and M65 signals in blood samples of patients with advanced cancer. The antibody data were validated against quantitative RT-PCR. The same investigators also studied the BH-3 mimetic ABT-737 in mice bearing small-cell lung cancer xenografts [54]. Circulating levels of CK18 and caspase-cleaved CK18 were shown to correlate with tumour burden. ABT-737 caused increases in cleaved CK18 that subsequently declined. Cummings and colleagues also studied the aurora kinase inhibitor AZD1152 in mice with SW620 human colon tumour xenografts and concluded that M30 was a biomarker of AZD1152-induced apoptosis, while M65 was a biomarker of therapeutic response [55]. In patients with testicular cancer, M30 and M65 were shown to give a measure of cell death after treatment with bleomycin, etoposide, and cisplatin [46]. Olofsson et al. showed that the affinity of the M30 antibody for murine caspase fragments was relatively low, so that in mice carrying human tumour xenografts, the M30 response following drug treatment was primarily from the human tumour cells [56]. Dean et al. [57] studied CK18 and apoptosis biomarkers in small cell lung cancer (SCLC) lines in vitro, and in SCLC patients treated with obatoclax, an inhibitor of the antiapoptotic protein $\mathrm{Bcl}-2$ [57]. In preclinical studies, peak levels of apoptosis occurred 24 hours after obatoclax treatment. In the clinical study, all the patients classified as responders after two cycles of treatment showed significantly increased levels of CK18 and cleaved CK18 on day 3 of the study. Dive et al. [58] discussed the use of CK18 and cleaved CK18 as biomarkers for treatment of pancreatic cancer. The M65 levels were higher in patients with metastatic disease compared with locally advanced disease, which were in turn higher than in patients following resection. The baseline levels in patients with pancreatic cancer were affected by the presence of obstructive jaundice, but the authors concluded that clinical biomarker studies of serial CK18 levels gave useful information in pancreatic cancer, providing consideration was given to the potentially confounding factors.

Nonepithelial tumours, such as lymphomas, do not express CK18. Increases in circulating CK18 in lymphoma patients treated undergoing chemotherapy were attributed to epithelial toxicity [45]. Circulating nucleosomal DNA could be used as a PD biomarker in these patients [45].

Green and colleagues [59-61] measured apoptosis in cancer patients treated with the cyclin-dependent kinase (cdk) inhibitor, seliciclib. Seliciclib is known to induce apoptosis in sensitive tumours by inhibiting transcription of the mRNA for the antiapoptotic protein Mcl-1 [62]. Total CK18 was measured with the TPS antibody, and cleaved CK18 with the M30 antibody. Seliciclib induced an increase in circulating apoptotic markers in 50\% (20/40) of phase I cancer patients treated with. $800 \mathrm{mg}$ twice daily. Two patients with sarcomas, which do not express CK18, showed low baseline levels, and no increase after treatment. Signals given by the TPS and M30 antibodies were highly correlated. In patients where both PK and biomarkers were measured, the M30 score showed a significant correlation with seliciclib AUC [63]. In 43 phase I patients analyzed, the increase in both biomarkers following treatment correlated with seliciclib dose. In this phase I study, the biomarker data provided a suggestive indication that the higher doses of seliciclib were triggering the induction of apoptosis in these patients.

Ward et al. [47], reviewing the literature on serological biomarkers of apoptosis, emphasise that (in contrast with tissue biomarkers), serum biomarkers have the great advantage of allowing repeat sampling over time. They concluded that a single biomarker would often not have the power to predict proof of concept and patient outcome, but that the potential existed to do this using panels of multiple biomarkers, particularly if this could be done in small sample volumes using multiplex ELISA technologies.

\section{PK/PD Models of Apoptosis}

The foregoing discussion has demonstrated that the advantages of measuring drug-induced apoptosis by plasma biomarker assays are its wide applicability across many classes of anticancer drugs, its minimally invasive nature, the small sample volumes required, and the ability to sample at multiple time points. Another advantage of this approach which has not as yet been widely used is its potential for data modelling. Cummings et al. [64] examined alternative methods of data analysis for M30 and M65 assays, using the $\beta$-expectation tolerance interval as an endpoint for assessing assay accuracy. Lancashire et al. [65] used logistic regression, fractional polynomial regression, artificial neural networks (ANN), and support vector machines to derive predictive models for colorectal cancer, based upon a serum panel of insulin-like growth factor (IGF) peptides. Serum IGF-I, IGF-II, and IGF-binding proteins (IGFBP)-2 and -3 were measured. The greatest predictive power was achieved using ANN models.

The availability of an extensive data set of M30 and TPS apoptosis measurements from the phase I clinical trials of seliciclib made possible the development of a detailed PK/PD model [63]. The PD portion of the model was a description of apoptosis based upon the model of Hua et al. [23], modified to describe tumours that are immortalised by overexpression of the antiapoptotic protein Mcl-1. The PK portion of the model was based upon the model of de la Motte and Gianella-Borradori [66]. Tissue culture observations had shown that induction of apoptosis required concentrations to remain above a threshold level $(3-10 \mu \mathrm{M}$, depending upon the cell line) for a minimum period of time (typically 5-8 hr). If drug concentrations fell below the threshold before the minimum time had elapsed, no cell killing was observed, though reversible growth arrest was seen. This time-above-threshold approximation gave a rough fit to the clinical biomarker data, but the kinetics of apoptosis could be more accurately described by the PD model of apoptosis based upon the Hua model. The PD doseresponse curve predicted by this model was highly nonlinear 
with a dose threshold of $1000 \mathrm{mg}$ per patient for a single dose, or $800 \mathrm{mg}$ for repeat dosing [63]. Simulation of a dose of $1600 \mathrm{mg}$ every $12 \mathrm{hr}$ for 30020days predicted sharp peaks in plasma concentration, each of which was followed several hours later by a wave of apoptosis. Divided dosing was predicted to require a higher total daily dose to induce apoptosis than a single daily dose. Divided doses gave steeper dose-response curves than single daily doses, and the curves for once, twice, and three-times daily treatment converged to a single asymptote [63].

One advantage of mechanism-based PD models is that they facilitate "thought experiments" of hypothetical situations. For example, the question was posed: if a tumour cell is immortalised by upregulation of Bcl-2, how may this affect response to seliciclib? The model predicted that such cells would be much less sensitive in vivo to seliciclib. The reason appears to be that $\mathrm{Bcl}-2$ transcripts turn over much more slowly than Mcl-1 transcripts, making Bcl-2 overproducing cells relatively stable to temporary inhibition of RNA transcription (unpublished simulations of the author).

\section{Functional Imaging}

The technique of functional imaging using nuclear magnetic resonance (MRI) or positron emission tomography (PET) has revolutionised neuroscience by making it possible to correlate metabolic activity in different parts of the brain with mental states in conscious humans. The potential exists for imaging to have an equally profound effect on PD of anticancer drugs. By devising appropriate reagents, it is possible to visualise drug effects, as they happen, within tumour and normal tissues. The first published study of this kind [67] exploited the depletion of cellular thymidylate by the thymidylate synthase inhibitor AG337 (Thymitaq) to increase the fractional retention of [11C]-thymidine. The PET effect correlated closely with plasma deoxyuridine concentration. Other early PD studies with this noninvasive technology were reviewed by Workman et al. [68]. Chung et al. [69] used MRI to study PD effects of the HDAC inhibitor LAQ824. Beloueche-Babari et al. [70] discussedd several studies using MRI to monitor PD effects in preclinical systems with a number of drugs, including a choline kinase inhibitor, and a phase I clinical trial with the HSP90 inhibitor 17-AAG. Mitchell et al. [71] used MRI to visualise residual disease in patients following chemotherapy for recurrent ovarian cancer and showed that it correlated with the serological biomarker Ca125. McKinley et al. [72] used PET imaging with [18F]-fluorodeoxyglucose to demonstrate a PD effect of the IGF-1R inhibitor OSI-906 on glucose metabolism in preclinical mouse models of lung cancer.

These studies demonstrate the potential of functional imaging studies in pharmacodynamics. As yet, none of the PD imaging data thus generated have been fitted to PD models. The need for frequent repeat scanning may complicate the use of MRI and PET data in PD modelling, but the potential advantages of PD models based upon imaging data are so great that it is likely that such models will be published before too long.

\section{Biomarkers as Endpoints for Combination Chemotherapy Studies}

One application of PD modelling of biomarker data that shows great potential is the quantitation of combined drug effects in clinical trials. Currently, drug combinations are evaluated in preclinical models, and promising combinations are tested clinically using classical endpoints-tumour size, time to progression, or survival. These endpoints are able to determine in a qualitative way whether the activity of combinations is equivalent, superior, or inferior to that of the single agents, but it is usually not possible to quantify the drug interactions. PD biomarker endpoints can be analyzed so that combined drug effects on tumour, as well as on sensitive normal tissues, can be determined. Greco and Jackson [73] showed that biomarker effects on tumour cells and normal cells in vitro could be combined with PK data to predict in vivo or clinical drug interactions from in vitro data by PK/PD modelling. Iadevaia et al. [33] used a computational procedure that integrated mass action modelling of phospho-proteomics with particle swarm optimization to predict optimal combinations of inhibitors of the IGF-1 signalling network in a breast cancer cell line.

For many drug combinations, activity is highly dependent on the order in which the drugs are administered. Orrell et al. [74, 75] used PD modelling of biomarker data to predict the sequence-dependence of a drug combination. Their virtual tumour combined PK data, biomarkers, cell cycle kinetics, and a three-dimensional structure in which the central core of the tumour was necrotic. Their simulations were validated against xenografts, and the model correctly predicted the different outcomes of simultaneous and sequential administration of a two-drug combination.

\section{Conclusions: Twelve Things You Can Do with a PK/PD Model}

The recent developments in characterization and validation of PD biomarkers of anticancer drug action have greatly increased the range and predictive power of PD modelling, particularly in conjunction with PK. Promising applications include the following.

(1) Predicting optimal drug doses and routes of administration. PK models can predict plasma (or tissue) concentrations, but where drug selectivity is an issue, which is usually the case in oncology, a PK/PD model will probably be more predictive.

(2) Predicting optimal scheduling of multiple doses where drug effects lag behind drug concentrations. Optimal scheduling will often depend upon the time course of a PD effect, as well as on PK, so a predictive model must describe both.

(3) Relating efficacy biomarker responses to clinical outcome, and determining the comparative information content of alternative biomarkers in the prediction of clinical efficacy. Preclinical data can be used to relate degrees of biomarker response to antitumour 
responses, and the correlation can be extrapolated to the clinical situation.

(4) Predicting optimal protocols for tumours of different cytokinetic properties (e.g., doubling time, cell loss factor, quiescent fraction), and extrapolating from human tumour responses in murine xenograft models, to the clinical situation where cytokinetic parameters are often different.

(5) Predicting the impact of particular levels of drug resistance on clinical outcome and prediction of optimal treatment strategies for tumours that have developed partial drug resistance.

(6) Predicting when to change the treatment regimen to minimise or delay the onset of drug resistance.

(7) Relating toxicity biomarker responses to tolerability. If biomarkers for both efficacy and toxicity are available, the comparative selectivity of different regimens may be predicted.

(8) For a drug with multiple sites of action, PK/PD models can be used to explore the relationship of the different mechanisms to efficacy and toxicity.

(9) PK/PD modelling of biomarker data can predict the effects of drug combinations (additivity, synergism, antagonism) and optimal combination protocol design, for drugs that have metabolic or cytokinetic interactions.

(10) Predicting dose and schedule for a particular toxicity cut-off. By using population PK/PD data we may make predictions about what proportion of a treatment group may be expected to have particular levels of response (or particular levels of adverse events) for a given treatment regimen.

(11) Predicting comparative advantages of alternative clinical development strategies. PK/PD models can form the basis of virtual clinical trial software, making it possible to compare multiple possible trial designs in silico, before committing resources to the preferred study design [76-79].

(12) PK/PD models may be used to develop a sampling strategy, that is, to predict how to time the sampling of plasma or other tissues to obtain maximum information from the minimum number of samples.

Finally, in the long term, it will be possible to use PD biomarker data, in the context of a validated PK/PD model, as a surrogate endpoint that can predict the efficacy of an experimental treatment without the need to wait months or years for a clinical endpoint. This has become routine in other clinical areas (e.g., atherosclerosis), but the complexity and heterogeneity of malignant disease has so far meant that surrogate endpoints have not had sufficient predictive power. This will change as improved PD biomarkers are developed and validated, and as PK/PD modelling of these biomarker endpoints becomes an accepted drug development tool.

\section{Abbreviations}

ANN: Artificial neural network

AUC: Area under the concentration-time curve

cdk: Cyclin-dependent kinase

CK18: Cytokeratin-18

Gy: $\quad$ Gray (radiation dose unit)

HDAC: Histone deacetylase

HSP90: Heat shock protein 90

IGF: Insulin-like growth factor

IGFBF: Insulin-like growth factor binding protein

MRI: Magnetic resonance imaging

MTD: Maximum tolerated dose

PARP: Poly-(ADP-ribose) polymerase

PET: $\quad$ Positron emission tomography

PD: Pharmacodynamic

PK: $\quad$ Pharmacokinetic

SAC: Spindle assembly checkpoint

SCLC: Small cell lung cancer

TPS: Tissue polypeptide-specific antigen

TUNEL: Terminal dUTP nick end labelling

VEGF: Vascular endothelial growth factor.

\section{References}

[1] D. Sarker and P. Workman, "Pharmacodynamic biomarkers for molecular cancer therapeutics," Advances in Cancer Research, vol. 96, pp. 213-268, 2006.

[2] D. S. W. Tan, G. V. Thomas, M. D. Garrett et al., "Biomarkerdriven early clinical trials in oncology: a paradigm shift in drug development," Cancer Journal, vol. 15, no. 5, pp. 406420, 2009.

[3] Q. Zhou and J. M. Gallo, "The pharmacokinetic/ pharmacodynamic pipeline: translating anticancer drug pharmacology to the clinic," AAPS Journal, vol. 13, no. 1, pp. 111-120, 2011.

[4] R. J. Keizer, J. H. M. Schellens, J. H. Beijnen, and A. D. R. Huitema, "Pharmacodynamic biomarkers in model-based drug development in oncology," Current Clinical Pharmacology, vol. 6, no. 1, pp. 30-40, 2011.

[5] A. Sharma and W. J. Jusko, "Characterization of four basic models of indirect pharmacodynamic responses," Journal of Pharmacokinetics and Biopharmaceutics, vol. 24, no. 6, pp. 611-635, 1996.

[6] R. C. Jackson, Computer Techniques in Preclinical and Clinical Drug Development, CRC Press, Boca Raton, Fla, USA, 1996.

[7] I. Rafi, G. A. Taylor, J. A. Calvete et al., "Clinical pharmacokinetic and pharmacodynamic studies with the nonclassical antifolate thymidylate synthase inhibitor 3,4-dihydro-2amino-6-methyl-4-oxo-5-(4-pyridylthio)-quinazolone dihydrochloride (AG337) given by 24-hour continuous intravenous infusion," Clinical Cancer Research, vol. 1, no. 11, pp. 1275-1284, 1995.

[8] J. E. Kalns, N. J. Millenbaugh, M. G. Wientjes, and J. L. S. Au, "Design and analysis of in vitro antitumor pharmacodynamic studies," in Proceedings of the American Association for Cancer Research, vol. 36, 1995, abstract no. 279.

[9] P. Jordan and R. Gieschke, "Explicit solutions for a class of indirect pharmacodynamic response models," Computer Methods and Programs in Biomedicine, vol. 77, no. 2, pp. 9197, 2005. 
[10] E. F. Choo, M. Belvin, J. Chan et al., "Preclinical disposition and pharmacokinetics-pharmacodynamic modeling of biomarker response and tumour growth inhibition in xenograft mouse models of G-573, a MEK inhibitor," Xenobiotica, vol. 40, no. 11, pp. 751-762, 2010.

[11] D. P. Lane, "Oncogenic intelligence: cell immortalization and transformation by the p53 gene," Nature, vol. 312, no. 5995, pp. 596-597, 1984.

[12] B. D. Aguda and Y. Tang, "The kinetic origins of the restriction point in the mammalian cell cycle," Cell Proliferation, vol. 32, no. 5, pp. 321-335, 1999.

[13] P. Duesberg, R. Li, A. Fabarius, and R. Hehlmann, "Aneuploidy and cancer: from correlation to causation," Contributions to Microbiology, vol. 13, pp. 16-44, 2006.

[14] F. Lévi, A. Altinok, and A. Goldbeter, "Circadian rhythms and cancer chronotherapeutics," in Cancer Systems Biology, Bioinformatics and Medicine, A. Cesario and F. Marcus, Eds., pp. 381-407, Springer, Dordrecht, The Netherlands, 2011.

[15] B. Novák and J. J. Tyson, "A model for restriction point control of the mammalian cell cycle," Journal of Theoretical Biology, vol. 230, no. 4, pp. 563-579, 2004.

[16] C. Chassagnole, R. C. Jackson, N. Hussain et al., "Using a mammalian cell cycle simulation to interpret differential kinase inhibition in anti-tumour pharmaceutical development," BioSystems, vol. 83, no. 2-3, pp. 91-97, 2006.

[17] C. Gérard and A. Goldbeter, "Temporal self-organization of the cyclin/Cdk network driving the mammalian cell cycle," Proceedings of the National Academy of Sciences of the United States of America, vol. 106, no. 51, pp. 21643-21648, 2009.

[18] D. Gilbert, H. Fuß, X. Gu et al., "Computational methodologies for modelling, analysis and simulation of signalling networks," Briefings in Bioinformatics, vol. 7, no. 4, pp. 339353, 2006.

[19] R. P. Araujo, E. F. Petricoin, and L. A. Liotta, "A mathematical model of combination therapy using the EGFR signaling network," BioSystems, vol. 80, no. 1, pp. 57-69, 2005.

[20] F. A. Brightman and D. A. Fell, "Differential feedback regulation of the MAPK cascade underlies the quantitative differences in EGF and NGF signalling in PC12 cells," FEBS Letters, vol. 482, no. 3, pp. 169-174, 2000.

[21] S. Y. Shin, O. Rath, A. Zebisch, S. M. Choo, W. Kolch, and K. H. Cho, "Functional roles of multiple feedback loops in extracellular signal-regulated kinase and Wnt signaling pathways that regulate epithelial-mesenchymal transition," Cancer Research, vol. 70, no. 17, pp. 6715-6724, 2010.

[22] M. Fussenegger, J. E. Bailey, and J. Varner, "A mathematical model of caspase function in apoptosis," Nature Biotechnology, vol. 18, no. 7, pp. 768-774, 2000.

[23] F. Hua, M. G. Cornejo, M. H. Cardone, C. L. Stokes, and D. A. Lauffenburger, "Effects of Bcl-2 levels on fas signaling-induced caspase-3 activation: molecular genetic tests of computational model predictions," Journal of Immunology, vol. 175, no. 2, pp. 985-995, 2005.

[24] M. Bentele, I. Lavrik, M. Ulrich et al., "Mathematical modeling reveals threshold mechanism in CD95-induced apoptosis," Journal of Cell Biology, vol. 166, no. 6, pp. 839-851, 2004.

[25] M. A. J. Chaplain and A. R. A. Anderson, "Mathematical modelling, simulation and prediction of tumour-induced angiogenesis," Invasion and Metastasis, vol. 16, no. 4-5, pp. 222-234, 1996.

[26] R. C. Jackson, Theoretical Foundations of Cancer Chemotherapy Introduced by Computer Models, Academic Press, San Diego, Calif, USA, 1992.
[27] T. Eissing, L. Kuepfer, C. Becker et al., "A computational systems biology software platform for multiscale modelling and simulation: integrating whole-body physiology, disease biology, and molecular reaction networks," Frontiers in Physiology, vol. 2, article 4, 2011.

[28] R. C. Jackson, "Predictive software for drug design and development: recent progress and future developments," Pharmaceutical Development \& Regulation, vol. 1, pp. 159-168, 2003.

[29] B. Basse, B. C. Baguley, E. S. Marshall et al., "Modelling cell death in human tumour cell lines exposed to the anticancer drug paclitaxel," Journal of Mathematical Biology, vol. 49, no. 4, pp. 329-357, 2004.

[30] B. Basse, W. R. Joseph, E. S. Marshall, and B. C. Baguley, "Analysis of radiation-induced changes to human melanoma cultures using a mathematical model," Cell Proliferation, vol. 43, no. 2, pp. 139-146, 2010.

[31] J. M. Hou, A. Greystoke, L. Lancashire et al., "Evaluation of circulating tumor cells and serological cell death biomarkers in small cell lung cancer patients undergoing chemotherapy," American Journal of Pathology, vol. 175, no. 2, pp. 808-816, 2009.

[32] W. S. Hsieh, B. K. Peh, T. Loh et al., "Pharmacodynamic effects of seliciclib (R-roscovitine, CYC202) in patients with undifferentiated nasopharyngeal carcinoma (NPC) using a window trial design," in EORTC-NCI-AACR International Conference on Molecular Targets and Cancer Therapeutics, 2006, abstract no. 279.

[33] S. Iadevaia, Y. Lu, F. C. Morales, G. B. Mills, and P. T. Ram, "Identification of optimal drug combinations targeting cellular networks: integrating phospho-proteomics and computational network analysis," Cancer Research, vol. 70, no. 17, pp. 6704-6714, 2010.

[34] F. R. Luo, Z. Yang, A. Camuso et al., "Dasatinib (BMS354825) pharmacokinetics and pharmacodynamic biomarkers in animal models predict optimal clinical exposure," Clinical Cancer Research, vol. 12, no. 23, pp. 7180-7186, 2006.

[35] S. Yamazaki, J. Skaptason, D. Romero et al., "Pharmacokinetic-pharmacodynamic modeling of biomarker response and tumor growth inhibition to an orally available cMet kinase inhibitor in human tumor xenograft mouse models," Drug Metabolism and Disposition, vol. 36, no. 7, pp. 1267-1274, 2008.

[36] L. Lee, H. Niu, R. Rueger et al., "The safety, tolerability, pharmacokinetics, and pharmacodynamics of single oral doses of CH4987655 in healthy volunteers: target suppression using a biomarker," Clinical Cancer Research, vol. 15, no. 23, pp. 7368-7374, 2009.

[37] L. Salphati, H. Wong, M. Belvin et al., "Pharmacokineticpharmacodynamic modelling of tumor growth inhibition and biomarker modulation by the novel phosphoinositol 3-kinase inhibitor GDC-0941," Drug Metabolism and Disposition, vol. 38, pp. 1436-1442, 2010.

[38] H. B. Mistry, R. C. Jackson, M. A. J. Chaplain, and F. A. Davidson, "A pharmacodynamic model of the mitotic spindle checkpoint and the action of aurora kinase inhibitors," in Proceedings of the 99th Annual Meeting of the American Association for Cancer Research (AACR '08), San Diego, Calif, USA, April 2008, abstract no. 1642.

[39] C. Chassagnole, E. Fernandez, F. Scaerou et al., "Systems biology analysis of a novel aurora kinase inhibitor: CYC116," in Proceedings of the Proceedings of the American Association for Cancer Research, 2008, abstract no. 1645. 
[40] H. B. Mistry, D. E. MacCallum, R. C. Jackson, M. A. J. Chaplain, and F. A. Davidson, "Modeling the temporal evolution of the spindle assembly checkpoint and role of Aurora B kinase," Proceedings of the National Academy of Sciences of the United States of America, vol. 105, no. 51, pp. 20215-20220, 2008.

[41] M. Hajduch, D. Vydra, P. Dzubak, M. Dziechciarkova, I. Stuart, and D. Zheleva, "In vivo mode of action of CYC116, a novel small molecule inhibitor of aurora kinases and VEGFR2," in Proceedings of the American Association for Cancer Research, 2008, abstract no. 5645.

[42] H. B. Mistry, D. E. MacCallum, R. C. Jackson, M. A. Chaplain, and F. A. Davidson, "A pharmacodynamic model of Aurora kinase inhibitors in the spindle assembly checkpoint," Frontiers in Bioscience, vol. 15, pp. 249-258, 2010.

[43] H. Kamei, R. C. Jackson, D. Zheleva, and F. A. Davidson, "An integrated pharmacokinetic-pharmacodynamic model for an Aurora kinase inhibitor," Journal of Pharmacokinetics and Pharmacodynamics, vol. 37, no. 4, pp. 407-434, 2010.

[44] A. Lindauer, P. Di Gion, F. Kanefendt et al., "Pharmacokinetic/pharmacodynamic modelling of biomarker response to sunitinib in healthy volunteers," Clinical Pharmacology and Therapeutics, vol. 87, pp. 601-608, 2010.

[45] A. Greystoke, J. P. B. O’Connor, K. Linton et al., “Assessment of circulating biomarkers for potential pharmacodynamic utility in patients with lymphoma," British Journal of Cancer, vol. 104, no. 4, pp. 719-725, 2011.

[46] E. C. de Haas, A. Di Pietro, K. L. Simpson et al., "Clinical evaluation of M30 and M65 ELISA cell death assays as circulating biomakers in a drug-sensitive tumor, testicular cancer," Neoplasia, vol. 10, no. 10, pp. 1041-1048, 2008.

[47] T. H. Ward, J. Cummings, E. Dean et al., "Biomarkers of apoptosis," British Journal of Cancer, vol. 99, no. 6, pp. 841846,2008

[48] S. Linder, A. M. Havelka, T. Ueno, and M. C. Shoshan, "Determining tumor apoptosis and necrosis in patient serum using cytokeratin 18 as a biomarker," Cancer Letters, vol. 214, no. 1, pp. 1-9, 2004.

[49] T. Ueno, M. Toi, and S. Linder, "Detection of epithelial cell death in the body by cytokeratin 18 measurement," Biomedicine and Pharmacotherapy, vol. 59, supplement 2, pp. S359-S362, 2005.

[50] S. Linder, "Cytokeratin markers come of age," Tumor Biology, vol. 28, no. 4, pp. 189-195, 2007.

[51] V. Barak, H. Goike, K. W. Panaretakis, and R. Einarsson, "Clinical utility of cytokeratins as tumor markers," Clinical Biochemistry, vol. 37, no. 7, pp. 529-540, 2004.

[52] S. Linder, M. H. Olofsson, R. Herrmann, and E. Ulukaya, "Utilization of cytokeratin-based biomarkers for pharmacodynamic studies," Expert Review of Molecular Diagnostics, vol. 10, no. 3, pp. 353-359, 2010.

[53] J. Cummings, M. Ranson, E. Lacasse et al., "Method validation and preliminary qualification of pharmacodynamic biomarkers employed to evaluate the clinical efficacy of an antisense compound (AEG35156) targeted to the X-linked inhibitor of apoptosis protein XIAP," British Journal of Cancer, vol. 95, no. 1, pp. 42-48, 2006.

[54] D. Micha, J. Cummings, A. Shoemaker et al., "Circulating biomarkers of cell death after treatment with the $\mathrm{BH}-3$ mimetic ABT-737 in a preclinical model of small-cell lung cancer," Clinical Cancer Research, vol. 14, no. 22, pp. 7304$7310,2008$.

[55] J. Cummings, C. Hodgkinson, R. Odedra et al., "Preclinical evaluation of M30 and M65 ELISAs as biomarkers of drug induced tumor cell death and antitumor activity," Molecular Cancer Therapeutics, vol. 7, no. 3, pp. 455-463, 2008.

[56] M. H. Olofsson, J. Cummings, W. Fayad et al., "Specific demonstration of drug-induced tumour cell apoptosis in human xenografts models using a plasma biomarker," Cancer Biomarkers, vol. 5, no. 3, pp. 117-125, 2009.

[57] E. J. Dean, J. Cummings, A. Roulston et al., "Optimization of circulating biomarkers of obatoclax-induced cell death in patients with small cell lung cancer," Neoplasia, vol. 13, no. 4, pp. 339-347, 2011.

[58] C. Dive, R. A. Smith, E. Garner et al., "Considerations for the use of plasma cytokeratin 18 as a biomarker in pancreatic cancer," British Journal of Cancer, vol. 102, no. 3, pp. 577-582, 2010.

[59] S. R. Green, S. Frame, I. Fleming et al., "Identification of pharmacodynamic markers to support clinical trials of CYC202 (R-roscovitine), a cyclin-dependent kinase inhibitor," in Proceedings of the American Association for Cancer Research, vol. 44, 2003, abstract no. 2967.

[60] S. R. Green, S. Frame, K. Watt et al., "Identification of clinically relevant pharmacodynamic biomarkers in patients treated with the cdk inhibitor CYC202 (R-roscovitine)," in EORTCNCI-AACR International Conference on Molecular Targets and Cancer Therapeutics, 2004, abstract no. 432.

[61] S. Frame, S. Anderson, K. Watt et al., "Identification and clinical validation of biomarkers to support the development of seliciclib (CYC202)," in Proceedings of the American Association for Cancer Research, vol. 46, 2005, abstract no. 460.

[62] D. E. MacCallum, J. Melville, S. Frame et al., "Seliciclib (CYC202, R-Roscovitine) induces cell death in multiple myeloma cells by inhibition of RNA polymerase IIdependent transcription and down-regulation of Mcl-1," Cancer Research, vol. 65, no. 12, pp. 5399-5407, 2005.

[63] R. C. Jackson, A. L. Barnett, S. J. McClue, and S. R. Green, "Seliciclib, a cell-cycle modulator that acts through the inhibition of cyclin-dependent kinases," Expert Opinion on Drug Discovery, vol. 3, no. 1, pp. 131-143, 2008.

[64] J. Cummings, C. Zhou, and C. Dive, "Application of the $\beta$ expectation tolerance interval to method validation of the M30 and M65 ELISA cell death biomarker assays," Journal of Chromatography B, vol. 879, no. 13-14, pp. 887-893, 2011.

[65] L. J. Lancashire, D. L. Roberts, C. Dive, and A. G. Renehan, "The development of composite circulating biomarker models for use in anticancer drug clinical development," International Journal of Cancer, vol. 128, no. 8, pp. 1843-1851, 2011.

[66] S. de la Motte and A. Gianella-Borradori, "Pharmacokinetic model of R-roscovitine and its metabolite in health male subjects," International Journal of Clinical Pharmacology and Therapeutics, vol. 42, no. 4, pp. 232-239, 2004.

[67] P. Wells, E. Aboagye, R. N. Gunn et al., "2-[11C]thymidine positron emission tomography as an indicator of thymidylate synthase inhibition in patients treated with AG337," Journal of the National Cancer Institute, vol. 95, no. 9, pp. 675-682, 2003.

[68] P. Workman, E. O. Aboagye, Y. L. Chung et al., "Minimally invasive Pharmacokinetic and Pharmacodynamic Technologies in hypothesis-testing clinical trials of innovative therapies," Journal of the National Cancer Institute, vol. 98, no. 9, pp. 580-598, 2006.

[69] Y. L. Chung, H. Troy, R. Kristeleit et al., "Noninvasive magnetic resonance spectroscopic pharmacodynamic markers of a novel histone deacetylase inhibitor, LAQ824, in human colon carcinoma cells and xenografts," Neoplasia, vol. 10, no. 4, pp. 303-313, 2008. 
[70] M. Beloueche-Babari, Y. L. Chung, N. M. S. Al-Saffar, M. Falck-Miniotis, and M. O. Leach, "Metabolic assessment of the action of targeted cancer therapeutics using magnetic resonance spectroscopy," British Journal of Cancer, vol. 102, no. 1, pp. 1-7, 2010.

[71] C. L. Mitchell, J. P. B. O'connor, A. Jackson et al., "Identification of early predictive imaging biomarkers and their relationship to serological angiogenic markers in patients with ovarian cancer with residual disease following cytotoxic therapy," Annals of Oncology, vol. 21, no. 10, pp. 1982-1989, 2010.

[72] E. T. McKinley, J. E. Bugaj, P. Zhao et al., "18FDG-PET predicts pharmacodynamic response to OSI-906, a dual IGF-1R/IR inhibitor, in preclinical mouse models of lung cancer," Clinical Cancer Research, vol. 17, no. 10, pp. 3332-3340, 2011.

[73] W. R. Greco and R. C. Jackson, "Prediction of the in vivo and clinical activity of drug combinations from in vitro data by differential pharmacodynamics," in Proceedings of the American Association for Cancer Research, 2008, abstract no. 4765.

[74] D. Orrell and E. Fernandez, "Using predictive mathematical models to optimise the scheduling of anti-cancer drugs," Innovations in Pharmaceutical Technology, no. 33, pp. 58-62, 2010.

[75] D. Orrell, E. Fernandez, D. Cronier et al., "Predicting the effect of combination schedules on xenograft tumor using the Virtual Tumor," in Proceedings of the American Association for Cancer Research, 2011, abstract no. 4918.

[76] P. Girard, M. Cucherat, and D. Guez, "Clinical trial simulation in drug development," Therapie, vol. 59, no. 3, pp. 287-304, 2004.

[77] C. Snell, D. Orrell, E. Fernandez, C. Chassagnole, and D. Fell, "Systems biology approaches to cancer drug development," in Cancer Systems Biology, Bioinformatics and Medicine, A. Cesario and F. Marcus, Eds., pp. 367-380, Springer, Dordrecht, The Netherlands, 2011.

[78] D. Ternant, E. Hénin, G. Cartron, M. Tod, G. Paintaud, and P. Girard, "Development of a drug-disease simulation model for rituximab in follicular non-Hodgkin's lymphoma," British Journal of Clinical Pharmacology, vol. 68, no. 4, pp. 561-573, 2009.

[79] S. Yamazaki, L. Nguyen, S. Vekich et al., "Pharmacokinetic/ pharmacodynamic response modelling of biomarker and tumor growth inhibition to an orally available heat shock protein 90 inhibitor in human tumor xenograft mouse model," Journal of Pharmacology and Experimental Therapeutics, vol. 338, no. 3, pp. 964-973, 2011. 

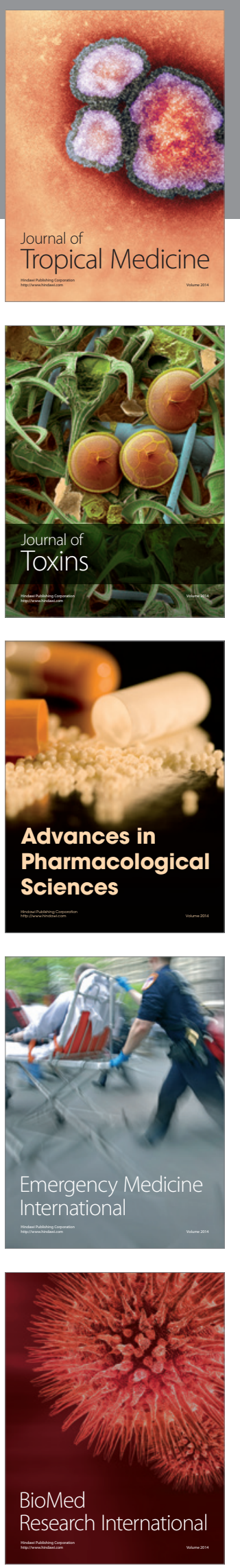
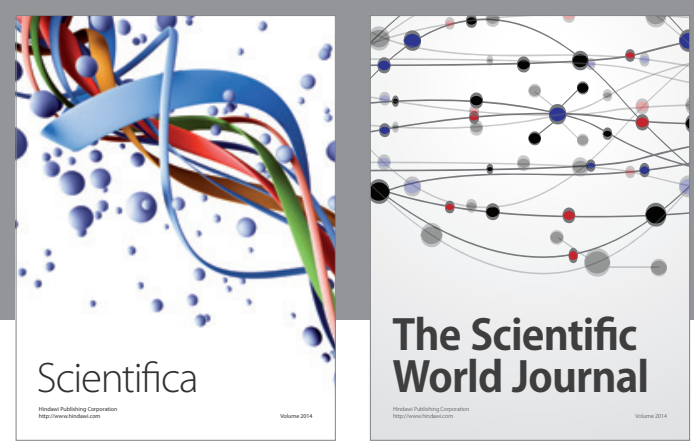

The Scientific World Journal
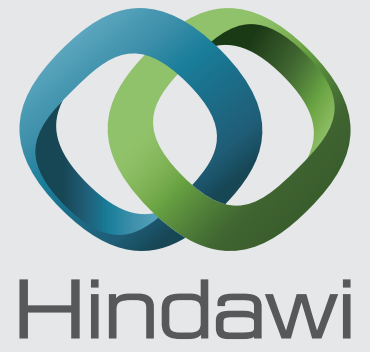

Submit your manuscripts at

http://www.hindawi.com
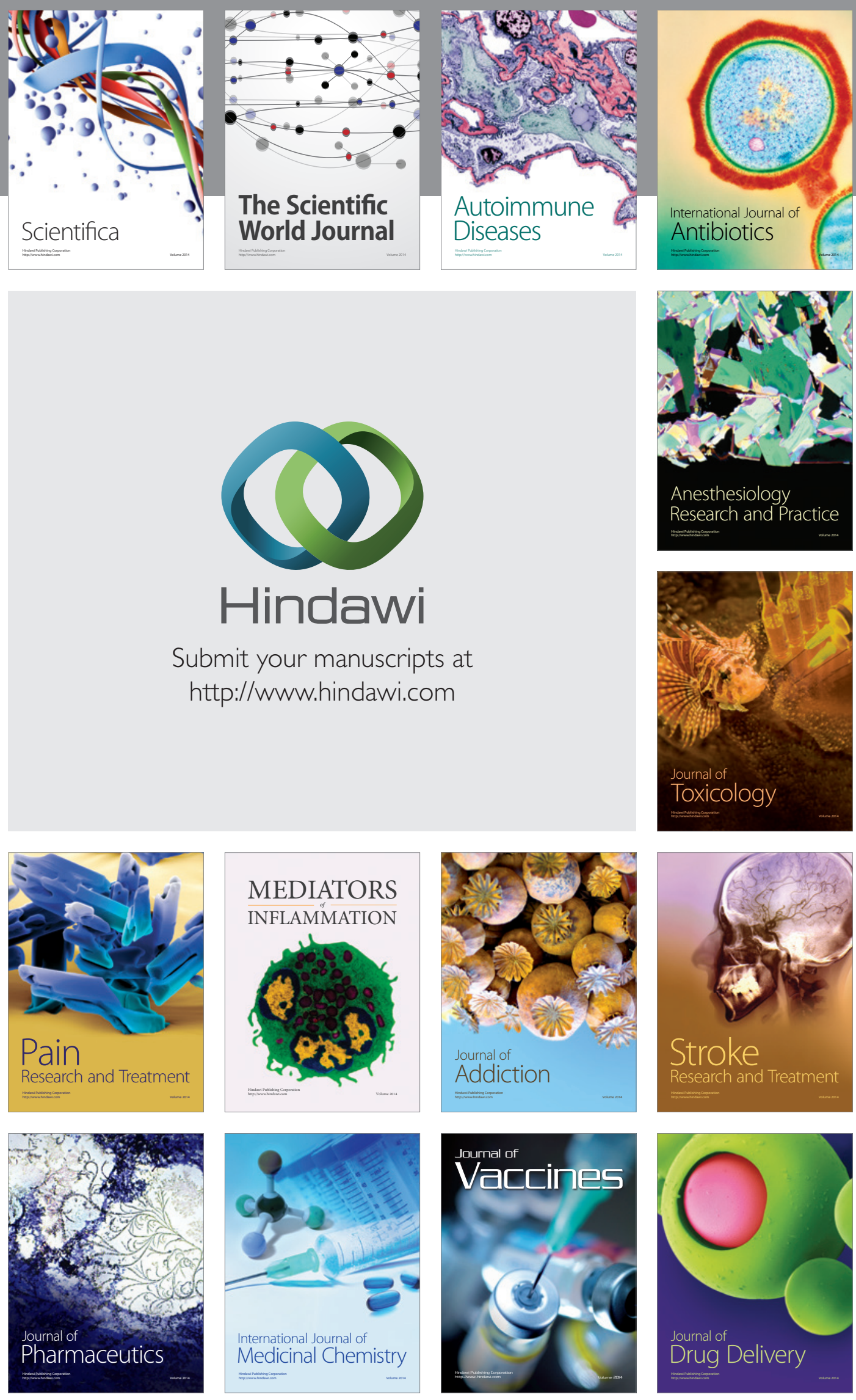